\title{
Prekanseröz lezyonların Helicobacter pylori eradikasyonuna olan etkisi
}

\author{
Precancerous lesions effects on the eradication of Helicobacter pylori
}

\author{
Evrim KAHRAMANOĞLU AKSOY', Muhammet Yener AKPINAR' ${ }^{1}$, Ferdane PIRINÇÇI SAPMAZ'1 Zeynep \\ GÖKTAŞ², Gülçin Güler ŞiMŞEK², Metin UZMAN', Yaşar NAZLIGÜL'
}

Sağlık Bilimleri Üniversitesi, Keçiören Eğitim ve Araştırma Hastanesi, ' Gastroenteroloji Bölümü, Ankara

Hacettepe Üniversitesi Sağlık Bilimleri Fakültesi, ${ }^{2}$ Beslenme ve Diyetetik Bölümü, Ankara

Sağlık Bilimleri Üniversitesi, Keçiören Eğitim ve Araştırma Hastanesi, ${ }^{3}$ Patoloji Bölümü, Ankara

\begin{abstract}
Giriş ve Amaç: Mide mukozasındaki Helicobacter pylori yoğunluğu, inflamasyonun aktivite derecesi, gastrit şiddeti ile eradikayon başarısı arasındaki ilişkiyi gösteren çalışma sayısı sınırlıdır. Biz çalışmamamızda; Helicobacter pylori eradikasyon başarısı üzerine gastrit şiddetinin ve Sidney klasifikasyon parametrelerinin etkisini araştırmayı amaçladık. Gereç ve Yöntem: Çalışmaya; Temmuz 2017 ve Aralık 2017 tarihleri arasında Keçiören Eğitim ve Araştırma Hastanesi'nde Helicobacter pylori enfeksiyonu tanısı patolojik olarak konmuş, eradikasyon için bizmut içeren standart dörtlü tedaviyi tamamlayan hastalar dahil edildi. Bulgular: Çalışmaya toplam 634 hasta dahil edildi. Antrumda atrofi varlığında Helicobacter pylori eradikasyon başarısı düşükken, intestinal metaplazi varlığında eradikasyon başarısı etkilenmemiştir (sırasıyla $p=0,025$ ve $p>0,05$ ); benzer şekilde korpustaki atrofi ve intestinal metaplazi olan grupta da eradikasyon başarısı daha azdı (sırasıyla $p=0,016$ ve $p=0,01$ ). Şiddetli gastrit gruplarında eradikasyon başarısızlığı daha fazlaydı (OLGA III-IV için $p=0.015$, OLGIM III-IV için p=0.032). Multiple lineer regresyon analizinde değerlendirildiğinde korpus intestinal metaplazi ve lenfoid agregasyon şiddeti bağımsız risk faktörü olarak değerlendirilmiştir (sırasıyla $p=0.002$ ve $p=0.042$ ). Sonuç: Gastrit ciddiyeti, intestinal metaplazi ve gastrik atrofi Helicobacter pylori eradikasyon tedavisi başarısı üzerinde negatif etki göstermektedir. Bu yüzden, patolojilerinde bu özelliklere sahip olan hastalarda tedavi protokolü ve tedavi süresi yeniden gözden geçirilmelidir.
\end{abstract}

Anahtar kelimeler: Atrofi, Helicobacter pylori, intestinal metaplazi

\section{Gíriş}

Helicobacter pylori ( $H$. pylori) enfeksiyonu; dünyada kronik gastrite yol açan en sık etkendir. Prevalansı gelişmekte olan ülkelerde \%80 civarında iken gelişmiş ülkelerde \%20 -50 arasında değişmektedir (1). Kronik gastritin yanı sıra; duodenal ülser, mide ülseri, mide adenokarsinoma, mide MALToma gibi birçok hastalığa yol açmaktadır (2). 1994 yıIında Uluslararası Kanser Araştırmaları Ajansı [(IARC) International Agency for Research on Cancer] tarafından mide kanseri için tip I kanserojen olarak sınıflandırılmıştır (3).

Kronik atrofik gastrit ve intestinal metaplazi; mide mukozasında gelişen prekanseröz değişiklikler olarak bilinmektedir. Mide mukozasında bu değişiklikleri gösteren
Background and Aims: There is limited data regarding the effect of gastritis severity, the existence of atrophy, and intestinal metaplasia on the Helicobacter pylori eradication. Herein we aimed to investigate the relationship between Helicobacter pylori eradication and histopathological parameters like gastritis severity, intestinal metaplasia and/or atrophy, and the Sydney classification. Material and Methods: Patients diagnosed with Helicobacter pylori gastritis were enrolled in the study between July 2017 and December 2017. All patients received standard quadruple therapy with bismuth salt. Results: In total, 634 patients were enrolled. The success of Helicobacter pylori eradication in the presence of atrophy in the gastric antrum was low, while the eradication success was not affected in the presence of intestinal metaplasia ( $p=0.025$ and $p>0.05$, respectively). Eradication success was lower in the group with atrophy and intestinal metaplasia in the antrum $(p=0.016$ and $p=0.01$, respectively). Eradication failure was higher in the group with severe gastritis ( $p=0.015$ for OLGA IIIIIV, $p=0.032$ for OLGIM III-IV). Intestinal metaplasia in the corpus and severity of the lymphoid aggregation were independent risk factors for treatment failure ( $p=0.002$ and $p=0.042$, respectively). Conclusion: Severity of gastritis, intestinal metaplasia, and gastric atrophy had a negative effect on Helicobacter pylori eradication success. Treatment protocols and duration should be carefully planned in patients with these features.

Key words: Atrophy, Helicobacter pylori, intestinal metaplasia

hastalar mide kanseri gelişimi açısından yüksek risk taşımaktadırlar. Kronik H. pylori enfeksiyonu prekanseröz histolojik değişikliklerin en önemli nedeni olarak bilinmektedir. H. pylori enfeksiyonunda; mide mukozasında çok sayıda aşamadan sonra kronik gastrit, atrofik gastrit ve intestinal metaplazi gelişir $(4,5)$. Atrofik gastrit ve intestinal metaplazi regresyonu; intestinal tip mide kanserinden korunmak için önemlidir. Her ne kadar bu konudaki çalışmaların sonucu çelişkili olsa da atrofik gastritin ve intestinal metaplazinin $H$. pylori eradikasyonundan sonra gerilediğini gösteren çalışmalar mevcuttur (6-8). 2012 ylında yayınlanan Avrupa Gastrointestinal Endoskopi Derneği 
(ESGE) kllavuzunda; H. pylori eradikasyonu ile atrofik gastritin parsiyel düzelebileceği; intestinal metaplazinin geriye dönüşünün olmayacağı ancak neoplastik progresyonun duraksayacağı belirtilmiştir (9).

Son yıllarda artan antibiyotik direncine bağlı olarak artan H. pylori eradikasyon başarısızlığı önemli bir sorun haline gelmektedir. Proton pompa inhibitörlerinin hızı metabolizmasına yol açan CYP2C19 ve MDR polimorfizmleri, sigara, obezite, diabetes mellitus, tedavi uyumsuzluğu eradikasyon başarısızlı̆ıına yol açan diğer nedenler olarak sıralanabilir (10-13). Mide mukozasındaki $H$. pylori yoğunluğu, inflamasyonun aktivite derecesi, gastrit şiddeti ile eradikayon başarısı arasındaki ilişkiyi gösteren çalışma sayısı sınırlıdır $(14,15)$. Biz çalışmamamızda; H. pylori eradikasyon başarısı üzerine gastrit şiddetinin ve Sidney klasifikasyon parametrelerinin etkisini araştırmayı amaçladık.

\section{GEREÇ ve YÖNTEM}

\section{Hastalar}

Çalışmaya; Temmuz 2017 ve Aralık 2017 tarihleri arasında Keçiören Eğitim ve Araştırma Hastanesi'nde non ülser dispepsi nedeni ile üst gastrointestinal sistem endoskopisi yapilıp $H$. pylori enfeksiyonu tanısı patolojik olarak konmuş, eradikasyon için bizmut içeren standart dörtlü tedaviyi tamamlayan ardışık 634 hasta dahil edildi.

\section{Tedavi Protokolü}

Tüm hastalar bizmut içeren standart dörtlü protokol ile tedavi edildi (14 gün boyunca bizmut subsalisilat $262 \mathrm{mg}$ q.i.d., rabeprazol $20 \mathrm{mg}$ b.i.d., tetrasiklin $500 \mathrm{md}$ q.i.d. ve metronidazol $500 \mathrm{mg}$ t.i.d.).

\section{$\boldsymbol{H}$. pylori Eradikasyon Kontrolü}

Tedavi bitiminden 4 hafta sonra gaitada H. pylori antijen testi yapıldı, negatif sonuç eradikasyon olarak tanımlandı. Gaitada antijen testi yapılmadan önce son 4 hafta içerisinde proton pompa inhibitörü, antibiyotik, bizmut subsalisilat, $\mathrm{H}_{2}$ blokörü kullanımına izin verilmedi. Hastalar eradikasyon başarısına göre; eradikasyon başarılı grup ve eradikasyon başarısız grup olarak ikiye ayrıldı.

\section{Histopatolojik Değerlendirme}

Üst gastrointestinal sistem endoskopisi sırasında antrum ve korpustan iki biyopsi örneği alındı. Preparatlar hematoksilen eozin ile boyandı, bütün preparatlar aynı patolog tarafından değerlendirildi. Gastrit aktivitesini, H. pylori yoğunluğunu, atrofi ve intestinal metaplaziyi derecelendirmek için güncellenmiş Sidney klasifikasyonu kullanıldı; $0=$ yok, 1 = hafif, 2 = orta, 3 = belirgin olarak değerlendirildi. Gastriti derecelendirmek için; uluslararası OLGA (operative link for gastritis assessment) değerlendirmesi, intestinal metaplaziyi derecelendirmek için OLGIM (operative link on gastric intestinal metaplasia assessment) değerlendirmesi kullanıldı (Evre 0-4). Evre 3 ve 4 OLGA ve OLGIM şiddetli gastrit olarak değerlendirildi.

\section{İstatistiksel Analiz}

Veriler SPSS IBM 22.0 (New York, ABD) programı kullanılarak analiz edilmiştir. Sayısal veriler ortalama ve standart sapma olarak belirtilmiştir. Nominal veriler sayı ve yüzde olarak belirtilmiştir. Sayısal veriler dağılım normalitesi incelenerek iki grup arası ortalamaların değerlendirilmesinde Student-t test kullanılmıştır. Kategorik verilerin analizinde Ki-kare testleri kullanılmıştır. H. pylori eradikasyon tedavisinin başarısız olmasına neden olan bağımsız değişkenlerin değerlendirilmesinde çoklu doğrusal regresyon modeli kullanılmışıı ve \%95 güven aralıkları (Cl) hesaplanmıştır. p değeri 0.05 'in altında istatistiksel olarak anlamlı sayılmıştır.

\section{BULGULAR}

\section{Demografik ve Klinik Özellikler}

Hastaların ortalama yaşı $46.3 \pm 14.18$ yıl idi. Hastaların \%40.9'u erkek \%59.1'i kadındı. 561 hastada eradikasyon sağlanırken 73 hastada eradikasyon sağlanamadı. Yaş, cinsiyet, vücut kitle indeksine göre eradikasyon başarısı açısından fark yokken ( $p>0.05$ ), sigara içmeyenlerde eradikasyon başarısı, içenlere oranla anlamlı oranda yüksekti $(p<0.001)$ (Tablo 1).

Tablo 1. Hastaların demografik özellikleri

\begin{tabular}{|c|c|c|c|c|}
\hline & $H p(-)(n=561)$ & $H p(+)(n=73)$ & p & Toplam $(n=634)$ \\
\hline Yaş (yıl) (Ortt_SS) & $45.9 \pm 14.21$ & $49.0 \pm 13.68$ & $p>0.05$ & $46.3 \pm 14.18$ \\
\hline BKI $(\mathrm{kg} / \mathrm{m} 2)(\mathrm{Ort} \pm \mathrm{SS})$ & $28.1 \pm 4.14$ & $28.1 \pm 5.89$ & $p>0.05$ & $28.1 \pm 4.37$ \\
\hline Cinsiyet \% (Erkek/Kadın) & $40.8 / 59.2$ & $41.1 / 58.9$ & $p>0.05$ & $40.9 / 59.1$ \\
\hline Sigara içme durumu \% (Evet/Hayır) & $20.7 / 79.3$ & $39.7 / 60.3$ & $p<0.001$ & 22.9/77.1 \\
\hline
\end{tabular}

BKi: Beden kitle indeksi. 
Tablo 2. Sidney klasifikasyon parametrelerine göre antrum ve korpus biyopsilerinde tedavi başarılı ve tedavi başarısız hastaların dağlıımı

\section{Antrum Biyopsi}

$\begin{array}{cccc}\text { Tedavi Başarılı } & \text { Tedavi Başarısız } & \text { Tedavi Başarılı } & \text { Tedavi Başarısız } \\ (n=561) & (n=73) & (n=561) & (n=73)\end{array}$

\section{H. pylori yoğunluğu}

$(-)$

$(+)$

$(++)$

$(+++)$

$\mathbf{P}$

\section{Inflamasyon}

$(-)$

$(+)$

$(++)$

$(+++)$

$\mathbf{P}$

\section{Aktivite}

$(-)$

$(+)$

$(++)$

$(+++)$

$\mathbf{P}$

\section{Atrofi}

$(-)$

$(+)$

$(++)$

$(+++)$

P

\section{Metaplazi}

$(-)$

$(+)$

$(++)$

$(+++)$

\section{$P$}

Lenfoid agregasyon/follikül

$(-)$

$(+)$

$(++)$

$(+++)$

P
$51(\% 9.1)$
$182(\% 32.4)$
$186(\% 33.2)$
$142(\% 25.3)$

$>0.05$

$52(\% 9.3)$
$80(\% 14.3)$
$251(\% 44.7)$
$178(\% 31.8)$

179 (\%31.9)

$130(\% 23.2)$

$187(\% 33.3)$

$65(\% 11.5)$

$>0.05$

$1(\% 1.4)$

$22(\% 30.1)$

$30(\% 41.1)$

$20(\% 27.4)$

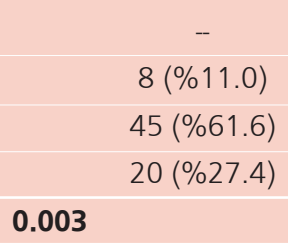

0.003

$>0.05$

$$
\begin{gathered}
524(\% 93.4) \\
24(\% 4.3) \\
6(\% 1.1) \\
7(\% 1.2)
\end{gathered}
$$

0.025

$\begin{array}{ccc}20(\% 27.4) & 215(\% 38.3) & 23(\% 31.5) \\ 18(\% 24.7) & 155(\% 27.6) & 21(\% 28.8) \\ 29(\% 39.7) & 149(\% 26.6) & 27(\% 37.0) \\ 6(\% 8.2) & 42(\% 7.5) & 2(\% 2.7)\end{array}$

$60(\% 82.2)$

7 (\%9.6)

$3(\% 4.1)$

$3(\% 4.1)$

$489(\% 87.2)$

$45(\% 8.0)$

$22(\% 3.9)$

$5(\% 0.9)$

$>0.05$

$$
\begin{gathered}
57(\% 78.2) \\
9(\% 12.3) \\
5(\% 6.8) \\
2(\% 2.7)
\end{gathered}
$$

406 (\%72.4)

$95(\% 16.9)$

$45(\% 8.0)$

$15(\% 2.7)$
$95(\% 16.9)$

$114(\% 20.3)$

247 (\%44.0)

105 (\%18.7)

$88(\% 15.7)$

185 (\%33.0)

187 (\%33.3)

101 (\%18.0)

$6(\% 8.2)$

$23(\% 31.5)$

24 (\%32.9)

$20(\% 27.4)$

$>0.05$

4 (\%5.5)

$16(\% 21.9)$

$42(\% 57.5)$

$11(\% 15.1)$

0.031

$62(\% 84.9)$

$6(\% 8.2)$

$5(\% 6.8)$

0.016

$3(\% 0.5)$

$18(\% 3.2)$

9 (\%1.6)

-

$56(\% 76.7)$

$8(\% 11.0)$

7 (\%9.6)

$2(\% 2.7)$

0.001

$60(\% 82.2)$

$12(\% 16.4)$

1 (\%1.4)

\begin{tabular}{|c|c|c|c|}
\hline & Tedavi Başarılı ( $n=561$ ) & Tedavi Başarısız (n=73) & $p$ \\
\hline OLGA III-IV (n, \%) & $12(2.2)$ & $4(5.4)$ & $p=0.015$ \\
\hline OLGIM III-IV (n, \%) & $16(2.8)$ & $8(11.0)$ & $p=0.032$ \\
\hline
\end{tabular}

$>0.05$
417 (\%74.3)

$100(\% 17.8)$

$40(\% 7.2)$

$4(\% 0.7)$
$>0.05$

Tablo 3. Tedavi başarısına göre şiddetli gastrit görülme durumu

OLGA: Operative link for gastritis assessment, OLGIM: Operative link on gastric intestinal metaplasia assessment. 


\section{Histopatolojik Bulgular ve Eradikasyon Oranları Arasındaki ílişki}

Antrum ve korpus biyopsileri Sidney klasifikasyon parametrelerine göre değerlendirilmiş ve detayları Tablo 2'te verilmiştir. Antral biyopsiler ele alındığında; eradikasyonu başarısız olan 73 hastanın 13'ünde atrofi, 16'sında intestinal metaplazi saptanmıştır [13/73 (\%17.8) vs. 37/561 (\%6.6)], [16/73 (\%21.8) vs. 72/561 (\%12.8)]. Bu bağlamda intestinal metaplazi varlığı eradikasyon oranlarını etkilemezken ( $p$ >0.05), gastrik atrofinin varlığı eradikasyon başarısını istatistiksel anlamlı oranda azaltmaktadır $(p=0.025)$. Korpus biyopsileri ele alındığında ise; hem gastrik atrofi [11/73 (\%15) vs. 30/561 (\%5.3)] ve hem intestinal metaplazi varlığının [17/73 (\%23.3) vs. 42/561 (\%7.5)] eradikasyon başarısını azalttığı izlendi ( $p=0.016$ ve $p=0.001$, sırasıyla). Şiddetli gastrit gruplarında eradikasyon başarısızlığı daha fazlaydı (OLGA III-IV için p=0.015, OLGIM III-IV için p=0.032) (Tablo 3). Multiple lineer regresyon analizinde sadece sigara içmek tedavi başarısızlığı açısından bağımsız risk faktörü olarak saptan$d_{\imath}(p<0.001)$ (Tablo 4). Antrum ve korpus biyopsilerinde Sidney klasifikasyon parametreleri multiple lineer regresyon analizinde değerlendirildiğinde korpus intestinal metaplazi varlığı tedavi başarısızığı açısından ve lenfoid agregasyon şiddetinin tedavi başarısı açısından bağımsız risk faktörü olarak değerlendirilmiştir ( $p=0.002$ ve $p=0.042$, sırasıyla) (Tablo 5).

Tablo 4. H. pylori eradikasyon başarısızlık oranının multiple lineer regresyon analizi ile değerlendirilmesi

\begin{tabular}{|lcccc}
\hline & B Katsayısı & Standart Hata $\beta$ & $\mathbf{p}$ & $\mathbf{C l , 9 5 \%}$ \\
\hline Yaş & 0.001 & 0.001 & 0.153 & $(-0.001-0.003)$ \\
\hline Cinsiyet (K) & -0.014 & 0.026 & 0.585 & $(-0.064-0.036)$ \\
\hline BKi & $<0.001$ & 0.003 & 0.897 & $(-0.006-0.005)$ \\
\hline Sigara içmek & 0.121 & 0.030 & $<0.001$ & $(0.062-0.180)$ \\
\hline Antrum H. pylori yoğunluğu & 0.014 & 0.015 & 0.347 & $(-0.015-0.043)$ \\
\hline Korpus H. pylori yoğunluğu & 0.023 & 0.014 & 0.113 & $(-0.005-0.051)$ \\
\hline OLGA & 0.037 & 0.027 & 0.169 & $(-0.016-0.090)$ \\
\hline OLGIM & 0.039 & 0.024 & 0.105 & $(-0.008-0.086)$ \\
\hline
\end{tabular}

BKI: Beden kitle indeksi, OLGA: Operative link for gastritis assessment, OLGIM: Operative link on gastric intestinal metaplasia assessment.

Tablo 5. H. pylori eradikasyon başarısızlık oranının antrum ve korpus biyopsi bulgularına göre multiple lineer regresyon analizi

\begin{tabular}{|lllll}
\hline & B Katsayısı & Standart Hata $\beta$ & p & Cl, 95\% \\
\hline Antrum biyopsi & & & & \\
\hline H. pylori yoğunluğu & 0.019 & 0.020 & 0.352 & $(-0.021-0.058)$ \\
\hline Inflamasyon & 0.022 & 0.021 & 0.288 & $(-0.019-0.063)$ \\
\hline Aktivite & -0.018 & 0.016 & 0.251 & $(-0.049-0.013)$ \\
\hline Atrofi & 0.053 & 0.115 & 0.647 & $(-0.174-0.280)$ \\
\hline Metaplazi & -0.034 & 0.064 & 0.600 & $(-0.160-0.092)$ \\
\hline Lenfoid agregasyon/follikül & -0.025 & 0.024 & 0.303 & $(-0.072-0.022)$ \\
\hline Korpus biyopsi & & & & $(-0.010-0.068)$ \\
\hline H. pylori yoğunluğu & 0.029 & 0.020 & 0.143 & $(-0.037-0.042)$ \\
\hline Inflamasyon & 0.003 & 0.020 & 0.898 & $(-0.030-0.042)$ \\
\hline Aktivite & 0.006 & 0.018 & 0.745 & $(-0.310-0.052)$ \\
\hline Atrofi & -0.129 & 0.092 & 0.162 & $(0.074-0.316)$ \\
\hline Metaplazi & 0.195 & 0.062 & 0.002 & $(-0.110--0.002)$ \\
\hline Lenfoid agregasyon/follikül & -0.056 & 0.028 & 0.042 & \\
\hline
\end{tabular}




\section{TARTIŞMA}

Biz çalışmamızda, gastrik atrofi ve intestinal metaplaziyi H. pylori eradikasyonunun başarısız olduğu grupta daha fazla bulduk. Yine eradikasyonun başarısız olduğu grupta şiddetli gastritin daha çok olduğunu saptadık (evre III-IV OLGA, OLGIM). Ayrıca sigara içimi, korpus biyopsisinde intestinal metaplazi şiddeti ve lenfoid agregasyonunun tedavi başarısızlı̆ı̆ üzerinde etkili olduğunu gösterdik.

H. pylori yoğunluğu ve inflamasyon şiddeti arasındaki ilişkiyi gösteren çok sayıda çalışma olmasına rağmen $H$. pylori yoğunluğu ve eradikasyon başarııı arasındaki ilişkiyi gösteren çalışma sonuçları çelişkilidir (16-18). Sheu ve ark. başlangıç $H$. pylori yoğunluğu ve serolojik titre yüksekliği fazla olan, üçlü eradikasyon tedavi alan hastalarda eradikasyon başarısının daha az olduğunu göstermişlerdir (15). Gümürdülü ve ark. çalışmalarında antral $H$. pylori yoğunluğunun eradikasyon başarısını etkilemediğini, korpus $H$. pylori yoğunluğunun eradikasyon başarısını negatif yönde etkilediğini göstermişlerdir (19). Yine Shah ve ark. H. pylori yoğunluğu ve eradikasyon başarısı arasında negatif korelasyon saptamışlardır (20).

Biz çalışmamızda H. pylori yoğunluğunun eradikasyon başarısı üzerine etkisini saptamadık. Unler ve ark. üçlü tedavi rejimi kullanan hastalarda bakteri yükünün eradikasyon başarısını etkilediğini göstermişler fakat standart dörtlü tedavi kullanan hastalarda böyle bir ilişki saptamamışlardır (21). Önal ve ark.nın çalışmasında da $H$. pylori yoğunluğu fazla olan hastalarda üçlü tedavi rejiminin başarısının daha az olduğu fakat bu durumun dörtlü tedavi rejimi alan grupta saptanmadığı belirtilmiştir (18). Yine aynı şekilde Kalkan ve ark.nın çalışmasında dörtlü tedavi rejimi kullanan hastalar değerlendirilmiş eradikasyon başarısı ile bakteri yoğunluğu arasında bir ilişki saptanmamıştır (22).

Mide kanserinin; mide mukozasının non-atrofik gastritten atrofik gastrit ve intestinal metaplazi, displazi ve sonunda karsinomaya yol açan sıralı değişikliklerin yol açtı̆ı çok aşamalı bir süreç olduğu düşünülmektedir. Bu sürecin $H$. pylori tarafından başlatılıp sürdürüldüğü düşünülmektedir. H. pylori eradikasyonunun gastrik atrofi ve intestinal metaplazi üzerine etkisi çeşitli çalışmalarda değerlendirilmiş olmasına rağmen bu mukozal değişikliklerin eradikasyon başarısı üzerine etkisini değerlendiren çalışma sayısı azdır $(4-6,23)$. Kamada ve ark. antral gastrik atrofinin $H$. pylori eradikasyon başarısına etkisini saptamazken Kalkan ve ark. gastrik atrofi ve intestinal metaplazi saptanan hastalarda $H$. pylori eradikasyon başarısını düşük olarak saptamışlardır. Yine evre III-IV OLGA ve evre III-IV OLGIM hastalarının eradikasyonun başarısız olduğu grupta daha çok olduğu saptanmıştır $(22,24)$. Biz de çalışmamızda H. pylori yoğunluğu ile eradikasyon başarısı arasında ilişki saptamazken eradikasyon başarısız grupta evre III-IV OLGA ve evre III-IV OLGIM hastalarını daha yüksek oranda saptadık. Literatürde $H$. pylori enfeksiyonu, lenfoid agregasyon varlığı ve şiddeti ile korele olarak saptanmıştır $(25,26)$. Biz çalışmamızda korpus intestinal metaplazi varlı̆ı̆ını eradikasyon başarısızlı̆ı açısından ve lenfoid agregasyon şiddetini literatürle çelişkili olarak eradikasyon başarısı açısından bağımsız risk faktörü olarak saptadık. Eradikasyon tedavi başarııını etkileyen faktörlerin değerlendirildiği Yang ve ark. ve Georgopoulos ve ark. da çalışmalarında bizim çalışmamızla farklı olarak başlangıç biyopsisinde lenfoid follikül varlığı ile eradikasyon başarısızlığı arasında ilişki saptamışlardır $(27,28)$. Literatürle çelişkili olan bu sonucu çalışmamızın retrospektif dizaynından kaynaklanmış olabileceğini ve bu konuda prospektif çalışmalara ihtiyaç duyulduğunu düşünüyoruz.

Sigara içime durumunu tedavi başarısızlığında bağımsız risk faktörü olarak saptadık, bu durum Suzuki ve ark.nın yayınladığı metaanalizle uyumlu idi (13).

Sonuç olarak biz çalışmamızda; standart dörtlü H. pylori eradikasyon tedavisi alan hastalarda sigara içimi ve korpusta intestinal metaplazi varlığını eradikasyon başarısızlığı üzerine bağımsız risk faktörü olarak saptadık. Gatrik atrofi ve intestinal metaplazinin ileri evre OLGA ve OLGIM varlığının bağımsız risk faktörü olmasa da eradikasyon başarısını olumsuz yönde etkileyebileceğini, H. pylori yoğunluğunun standart dörtlü tedavi alan hastalarda eradikasyon başarııını etkilemeyeceğini saptadık.

\section{KAYNAKLAR}

1. Go MF. Review article: natural history and epidemiology of Helicobacter pylori infection. Aliment Pharmacol Ther 2002;16(Suppl 1):3-15.

2. Sugano K, Tack J, Kuipers EJ, et al; faculty members of Kyoto Global Consensus Conference. Kyoto global consensus report on Helicobacter pylori gastritis. Gut 2015;64:1353-67.

3. Schistosomes, liver flukes and Helicobacter pylori. IARC Working Group on the Evaluation of Carcinogenic Risks to Humans. Lyon, 7-14 June 1994. IARC Monogr Eval Carcinog Risks Hum 1994;61:1-241.

4. Rugge M, Capelle LG, Cappellesso R, et al. Precancerous lesions in the stomach: from biology to clinical patient management.Best Pract Res Clin Gastroenterol 2013;27:205-23. 
5. de Vries AC, van Grieken NC, Looman CW, et al. Gastric cancer risk in patients with premalignant gastric lesions: a nationwide cohort study in the Netherlands. Gastroenterology 2008;134:945-52.

6. Chen HN, Wang Zi Li X, Zhou ZG. Helicobacter pylori eradication cannot reduce the risk of gastric cancer in patients with intestinal metaplasia and dysplesia: evidence from a meta-analysis. Gastric Cancer 2016;19:166-75.

7. Lee YC, Chen TH, Chiu HM, et al. The benefit of mass eradication of Helicobacter pylori infection:a community based study of gastric cancer prevention. Gut 2013;62:676-82.

8. Hwang YJ, Kim N, Lee HS, et al. Reversibility of atrophic gastritis and intestinal metaplasia after Helicobacter pylori eradication - a prospective study for up to 10 years. Aliment Pharmacol Ther 2018;47:380-90

9. Dinis-Ribeiro M, Areia M, de Vries AC, et al; European Society of Gastrointestinal Endoscopy; European Helicobacter Study Group; European Society of Pathology; Sociedade Portuguesa de Endoscopia Digestiva. Management of precancerous conditions and lesions in the stomach (MAPS): guideline from the European Society of Gastrointestinal Endoscopy (ESGE), European Helicobacter Study Group (EHSG), European Society of Pathology (ESP), and the Sociedade Portuguesa de Endoscopia Digestiva (SPED). Endoscopy 2012;44:74-94

10. Malfertheiner P, Megraud F, O'Morain CA, et al; European Helicobacter and Microbiota Study Group and Consensus panel. Management of Helicobacter pylori infection-the Maastricht V/Florence Consensus Report. Gut 2017;66:6-30.

11. Furuta T, Sugimoto M, Shirai N, et al. Effect of MDR1 C3435T polymorphism on cure rates of Helicobacter pylori infection by triple therapy with lansoprazole, amoxicillin and clarithromycin in relation to CYP 2C19 genotypes and 23S rRNA genotypes of $H$. pylori. Aliment Pharmacol Ther 2007;26:693-703

12. Abdullahi M, Annibale B, Capoccia D, et al. The eradication of Helicobacter pylori is affected by body mass index (BMI). Obes Surg 2008;18:1450-4.

13. Suzuki T, Matsuo $K$, Ito $H$, et al. Smoking increases the treatment failure for Helicobacter pylori eradication. Am J Med 2006;119:21724.

14. Moshkowitz M, Konikoff FM, Peled Y, et al. High Helicobacter pylori numbers are associated with low eradication rate after triple therapy. Gut 1995;36:845-7.

15. Sheu BS, Yang HB, Su IJ, et al. Bacterial density of Helicobacter pylori predicts the success of triple therapy in bleeding duodenal ulcer. Gastrointest Endosc 1996;44:683-8.
16. Ghasemi Basir HR, Ghobakhlou M, Akbari P, et al. Correlation between the intensity of Helicobacter colonization and severity of gastritis. Gastroenterol Res Pract 2017;2017:8320496.

17. Ardakani AS, Mohammadizadeh F. The study of relationship between Helicobacter density in gastric mucosa. J Res Med Sci 2006;11:282.

18. Onal IK, Sokmensuer C, Onal ED, et al. Clinical and pathological features of nodular gastritis in adults. Turk J Med Sci 2009;39:71923.

19. Gumurdulu Y, Serin E, Ozer B, et al. Low eradication rate of Helicobacter pylori with triple 7-14 days and quadriple therapy in Turkey. World J Gastroenterol 2004;10:668-71.

20. Shah DK, Jain SS, Mohite A, et al. Effect of $H$. pylori density by histopathology on its complications and eradication therapy. Trop Gastroenterol 2015;36:101-6.

21. Unler GK, Ozgur GT, Gokturk HS, et al. Does the urea breath test predict eradication of Helicobacter pylori infection?Acta Gastroenterol Belg 2016;79:3-7.

22. Kalkan IH, Sapmaz F, Güliter S, Atasoy P. Severe gastritis decreases success rate of Helicobacter pylori eradication. Wien Klin Wochenschr 2016;128:329-34.

23. Liu KS, Wong IO, Leung WK. Helicobacter pylori associated gastric intestinal metaplasia: Treatment and surveillance. World J Gastroenterol 2016;22:1311-20.

24. Kamada T, Hata J, Sugiu K, et al. Clinical features of gastric cancer discovered after successful eradication of Helicobacter pylori: results from a 9-year prospective follow-up study in Japan. Aliment Pharmacol Ther 2005;21:1121-6.

25. Kara N, Urganci N, Kalyoncu D, Yılmaz B. The association between Helicobacter pylori gastritis and lymphoid aggregates, lymphoid follicles and intestinal metaplasia in gastric mucosa of children. J Paediatr Child Health 2014;50:605-9.

26. Öner Ri, Özdaş S. Histopathological findings in morbid obese patients undergoing laporoscopic sleeve gastrectomy: Does $\mathrm{H}$. pylori infection effective on pathological changes? Obes Surg 2018 Apr 16 [Epub ahead of print].

27. Yang HB, Sheu BS, Su IJ, et al. Clinical application of gastric histology to monitor treatment of dual therapy in $\mathrm{H}$. pylori eradication. Dig Dis Sci 1997;42:1835-40.

28. Georgopoulos SD, Ladas SD, Karatapanis S, et al. Factors that may affect treatment outcome of triple Helicobacter pylori eradication therapy with omeprazole, amoxicillin, and clarithromycin. Dig Dis Sci 2000;45:63-7. 